\title{
Comparative Study and Simulation of TCP and UDP Traffic over Hybrid Network with Mobile IP
}

\author{
Purnya Awasthi \\ Kanpur Institute of Technology \\ Gautam Buddha Technical \\ University
}

\author{
Akhilesh Kosta \\ Kanpur Institute of Technology \\ Gautam Buddha Technical \\ University
}

\begin{abstract}
The performance of the TCP and UDP protocol is evaluated by simulating them in a hybrid network using Mobile IP and compared their performances on the basis of some performance metrics.

MANET is a group of wireless nodes connected through wireless link where nodes are free from any fixed infrastructure and each node maintains the routing information and act as router. It is also considered as secluded network because the nodes have no access to the outside world. But MANET integrated with internet can solve this problem by using an interconnected network where there can be two possible networks wired and wireless and communication between two different networks can be done by using Mobile IP. Mobile IP has been designed to allow the mobile node to use two IP addresses. One is the home address which remains unchanged and the other one care-of address changes at each new point of attachment.
\end{abstract}

\section{General Terms}

Hybrid Networks, Mobile IP

\section{Keywords}

TCP, UDP, Mobile IP, Hybrid networks, wired cum wireless network.

\section{INTRODUCTION}

Wireless communication technology is making immense progress and has become widely popular for access networks over past few years. These wireless access networks, such as Wireless Local Area Networks and cellular networks are usually connected to a wired backbone network. Although TCP and UDP are considered very reliable in wired networks and a lot of researches have been done on their behavior in wireless networks, there performance in hybrid networks is a matter of concern. Hybrid network has characteristics very different from those of wired networks and wireless networks [4].

Although many researches have been done to measure and analyze the TCP and UDP traffic over wireless networks or MANET but there was no comparison done for the hybrid networks with Mobile IP. NS2 is used as the simulation tool and studied various parameters in the hybrid environment to evaluate the behavior of two different transport layer protocols with Mobile IP being used as the protocol between two different networks.

\section{HYBRID NETWORK}

The hybrid networks are preferred instead of the pure wired networks and pure wireless ad hoc networks because the coverage area of the network can always be increased.

Hybrid network is a network consisting of both cellular base stations and ad hoc wireless nodes. The network used to simulate the Hybrid network contains 3 mobile nodes, 1 base station and 2 wired nodes. These three mobile nodes form the adhoc network as they are mobile and routing between them is

performed by DSDV protocol. Here data is exchanged between the mobile and non-mobile nodes.

The base station nodes are considered as the gateways between the wired and wireless nodes. They are connected to the wired nodes through a wired link. The base station node forwards all the packets from the wireless nodes destined to the wired nodes and vice a versa. So there is a need to keep the base station node

in the same domain as of the wireless nodes so that all the packets coming from wired nodes can be routed to their destination mobile nodes using the DSDV protocol [8].

\section{DSDV}

Destination sequenced distance vector routing (DSDV) is adapted from the conventional Routing Information Protocol (RIP) to ad hoc networks routing. DSDV is a routing protocol for ad hoc networks which was designed to overcome the looping problem of RIP and while keeping the simplicity of RIP.

In DSDV a new attribute is added named: sequence number, to each route table entry of the conventional RIP. By that added sequence number, the mobile nodes keep stale route information and the new route different and thus prevent the formation of routing loops.It is a table driven routing scheme based on Bellman-Ford algorithm.

\subsection{Packet Routing and Routing Table Management}

In DSDV, every mobile node has a routing table which has all available destinations to all other nodes, the metric, next hop and a sequence number generated by the destination node. The number is generated by the destination, and the emitter needs to send out the next update with this number.

Using such routing table stored in each mobile node, the packets are transmitted between the nodes of an ad hoc network. Each node of the ad hoc network either after a fixed duration of time or when some new routing information is needed to be propagated transmits the packets between the nodes of the adhoc network to update the routing information by multicasting or broadcasting. The packet send has a metric one which is used by the other nodes as they update their table 
and increase it by one every time it meets a new node and retransmit it to update other packets also. This process is repeated until all the nodes in the network receives a copy of the update packet with a corresponding metric. If any node receives multiple update packets for a same destination, the routes with more recent sequence numbers are always preferred. If the update packets have the same sequence number with the same node, the update packet with the smallest metric will be used.

\section{TCP}

Transport layer is made for process to process delivery, it deliver packets from one process to another. Transmission Control Protocol (TCP) is a connection oriented point-to-point protocol. It creates a virtual connection between two TCPs to send data. It also uses flow and error control mechanism at transport level. It is extensively used in the Internet.

\subsection{Reliability}

TCP is a reliable as it guarantees that all the packets that were send to the destination have reached and are in the order as they were sent.TCP takes care of this by using acknowledgements. Whenever a packet is received a acknowledgement is sent to the sender that packet is received. If the acknowledgement is not received by the sender it retransmits the packets after a time period maintained by the timer.

\subsection{Flow control}

In this mechanism, a window of segments (certain number of segments) is transmitted at once. Each segment has a sequence number. The receiver can acknowledge more than one segment at a time by acknowledging the highest one received, meaning that all the previous segments were successfully transmitted. And the size of the TCP window is informed to the sender by a field called the advertised window
(AWND) in the TCP header so that a fast sender does not overwhelm a slow receiver [5].

\subsection{Connection control}

To manage the dropped packets due to full buffer, a congestion window size is also maintained called CWND. The sending window is taken as being the minimum of the AWND and the CWND.

\subsection{Connection Establishment and Termination}

TCP connection starts with a three way handshake protocol. Firstly the receiver sends the SYN seq=x message to receiver. When the receiver receives the message it responds with a new sequence number $\mathrm{y}$ and acknowledgement message as SYN seq $=y, A C K x+1$. When the sender receives it, it acknowledges receiver with the ACK $y+1$ stating that acknowledgment is received. Thus sender and receiver perform a three way communication between them. In the first step a connection is established between sender and receiver then connection is maintained by the receiver until the sender receives the acknowledgement and releases the connection called as connection termination. This is also called as three way handshaking mechanism. This is done so that two nodes which are attempting to communicate, can exchange the parameters of the network TCP socket connection before transmitting data.

\subsection{Parameters that affect the performance of TCP in a hybrid environment}

The parameters are as follows:

\subsubsection{Bandwidth Limitation}

Wireless wide area networks offer limited raw bit rates which have to be shared between several users whereas wireless LAN offers sufficient bandwidth [14].

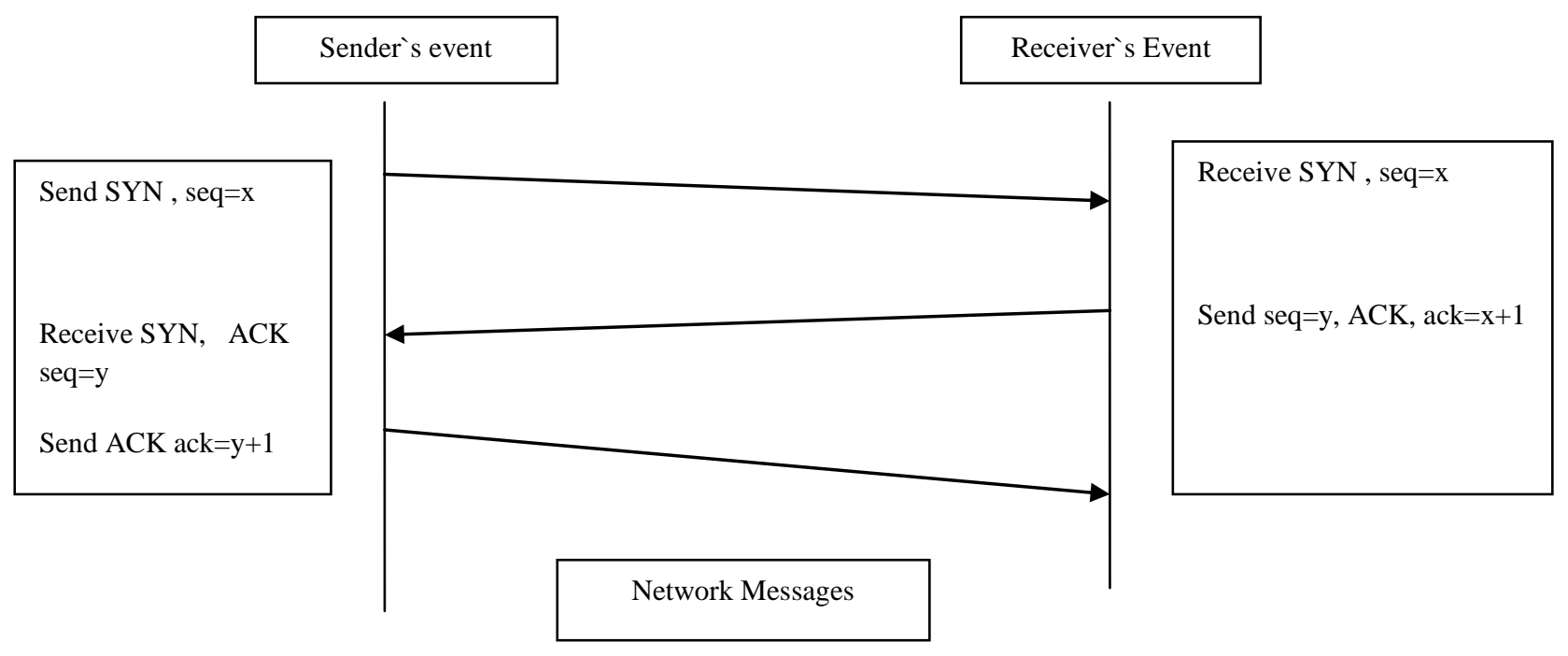

Figure 1. Three way handshake between TCP sender and receiver [7]. 


\subsubsection{Longer Latency Delay}

Wireless media exhibit longer latency delays than wired ones [13], this affects TCP throughput and increases the interactive delays perceived by the user [14].

\subsubsection{Vulnerable Transmission Losses}

Transmission losses are more in wireless media, so there is a need to find a solution which aims at alleviating this deficiency [14].

\subsubsection{User mobility}

Wireless networks enable the user to move around. hosts. When a host is moving from one cell to another handoff (or must be followed. During a handoff, all necessary information must be transferred between the two base stations so that the mobile host can continue to be connected[14].

TCP exhibits Limited bandwidth and longer latency delay in wireless networks and transmission errors are also more in wireless media, also when there are mobile nodes, the user mobility is a bigger concern. So there it would be beneficial to study the TCP behavior in a hybrid network rather than in wireless network or wired network as both have its pros and cons.

\section{USER DATAGRAM PRTOCOL}

User Datagram Protocol (UDP) is used as a transport layer protocol for transfer of data across Internet Protocol based network. The protocol uses datagrams to deliver the data. UDP is different in many ways with TCP. As TCP is connection oriented protocol where it set up a connection first then transfer data and the it terminate the connection, but in UDP there is no connection establishment between sender and receiver. The messages are broken into datagrams and send across the network. Each packet act as an individual message and is handled separately. Since each datagram has to keep more information than a TCP packet, the size of data it can carry is considerably low as compared to TCP. All the datagrams can follow any path to reach to the destination and hence the order in which they reach is not fixed. Neither the sender sends any acknowledgement to the sender that the datagram is received. Hence this protocol is considered as unreliable as it does not guarantee that the whole data reached to the destination or not.

While these were some demerits with UDP yet it is used in various applications where some data loss is tolerable like multimedia. Since in UDP there is no acknowledgement service hence the network has lower overhead and hence a better throughput and thus is faster than TCP which is suitable for the applications like multimedia.

\subsection{Performance of UDP in wireless networks}

For any closed wired network, the nature of the connections, the number of nodes and location of the recipients, what each needs to get from the transmission, and the response time of each node is UDP`s performance can be best.

Some researches have found that packet drop is more in UDP protocol as compared to TCP in a wireless network [15]. Transmission speed also more in UDP. The behavior of UDP in wired network and wireless network is mixed so there is a need to study the behavior of UDP in a hybrid network where it can show the mixed behavior.

\section{MOBILE IP}

Mobile IP was developer for meeting the solution of mobile users where there location is constantly changing and there is a need to stay connected to the internet all the time. Mobile IP helps in tracking hosts without a need to change their IP address[1].

\subsection{Working of Mobile IP}

Mobile IP can be demonstrated by its three major functions as AGENT DISCOVERY, REGISTRATION, TUNNELING

\subsubsection{Agent Discovery}

\subsubsection{Router advertisement}

Mobile IP agent advertisements are built on top of the Internet Control Message Protocol (ICMP) Router Discovery Protocol (IRDP). A beacon is sent after some defined interval of time by routers. By this beacon it advertises the services it supports. These beacons are either IP multicast or IP broadcast.

\subsubsection{Router solicitation}

Mobile agent solicitation is same as Router solicitation. In it a message is either multicast or broadcast to all other routers who listens this message can send their router advertisement. This is quicker as there is no wait for any periodic messages.

\subsubsection{Registration}

After discovery process, if the mobile nodes finds that it is in a foreign network, it singals its location updates to its home agent. This is done by mobile IP registration request. By this they convey to the Home Agent how to signal traffic to them by the Care Of Address. A registration reply is signaled either by the Home Agent or the Foreign Agent.

\subsubsection{Tunneling}

To send the routing information to the mobile node a logical link or a tunnel is set up which can carry IP packets. The home agent will intercept datagrams which are to send for the mobile node, and forward them to the mobile node. This is done by encapsulation.

The encapsulation process creates a logical construct called a tunnel between the device that encapsulates and the one that decapsulates [6].

\section{SIMULATION}

The simulation of the proposed network has been done by using Network Simulator-2. The network was simulated on the simulator as it supports the wired cum wireless network or the hybrid network.

A rectangular area of $600 \mathrm{~m}$ X $500 \mathrm{~m}$ was used for simulation. The simulation was carried using 1 mobile node, 2 wired nodes, 2 gateways and was run for 250 seconds. The source starts sending data and chooses one of the hosts as destination randomly. The pause time is varied starting from 0 sec., 10 sec., $20 \mathrm{sec}$, $30 \mathrm{sec}$., $40 \mathrm{sec}$., and $50 \mathrm{sec}$. (i.e. in the steps of $10 \mathrm{sec}$.) implemented respectively in a $100 \mathrm{~m}$. x $100 \mathrm{~m}$. The simulation time was taken to be of 100 seconds. 
Table 1: Simulation Parameter Values

\begin{tabular}{|c|c|}
\hline Transmitter range & $250 \mathrm{~m}$ \\
\hline Bandwidth & $2 \mathrm{Mbps}$ \\
\hline Simulation time & $50 \mathrm{sec}$ \\
\hline $\begin{array}{c}\text { Number of mobile } \\
\text { nodes }\end{array}$ & 1 \\
\hline $\begin{array}{c}\text { Number of Wired } \\
\text { nodes }\end{array}$ & 2 \\
\hline $\begin{array}{c}\text { Number of base } \\
\text { stations }\end{array}$ & 2 \\
\hline Max Speed & 20 \\
\hline Pause time & $10,20,30,40,50,60,70,80,90,100$ \\
\hline Terrain Area & $100 \mathrm{~m} . \times 100 \mathrm{~m} ., 1000 \mathrm{~m} . \mathrm{x} 1000 \mathrm{~m}$. \\
\hline Traffic type & Constant Bit Rate, FTP \\
\hline Packet size & 512 bytes \\
\hline MAC type & IEEE $802.11 \mathrm{~b}$ \\
\hline Antenna type & Omni-Antenna \\
\hline $\begin{array}{c}\text { Radio propagation } \\
\text { method }\end{array}$ & Two Ray Ground \\
\hline
\end{tabular}

\section{SIMULATION RESULTS AND ANALYSIS}

\subsection{Average end to end delay}

It's defined as time taken by a packet to reach from source to destination that is the time the packet is received minus the time it was generated at source.

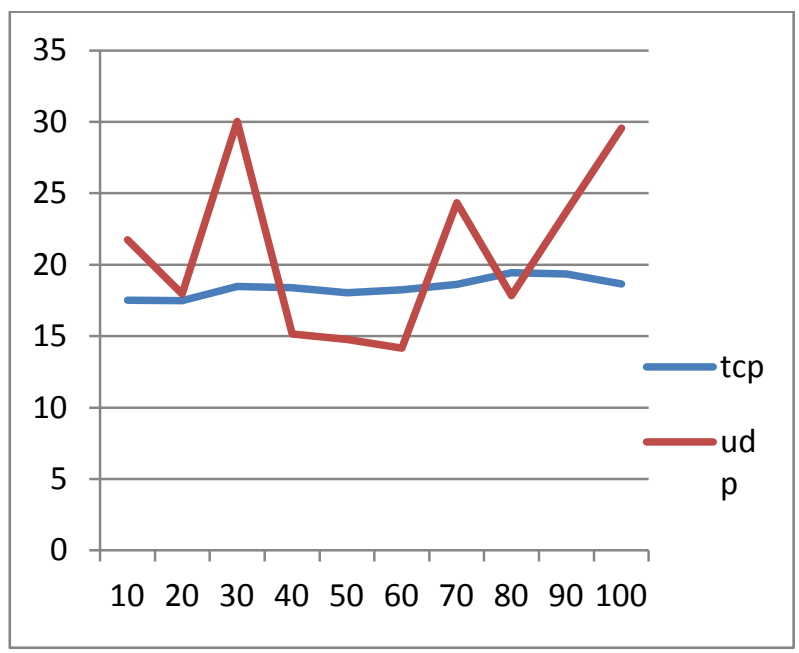

Figure 2: Average end to end delay versus pause time

This line graph illustrates the end to end delay over pause time by using TCP and UDP as the traffic modes

\subsection{Packet Delivery Ratio}

It is defined as the ratio of number of packets received to the total number of packet generated.

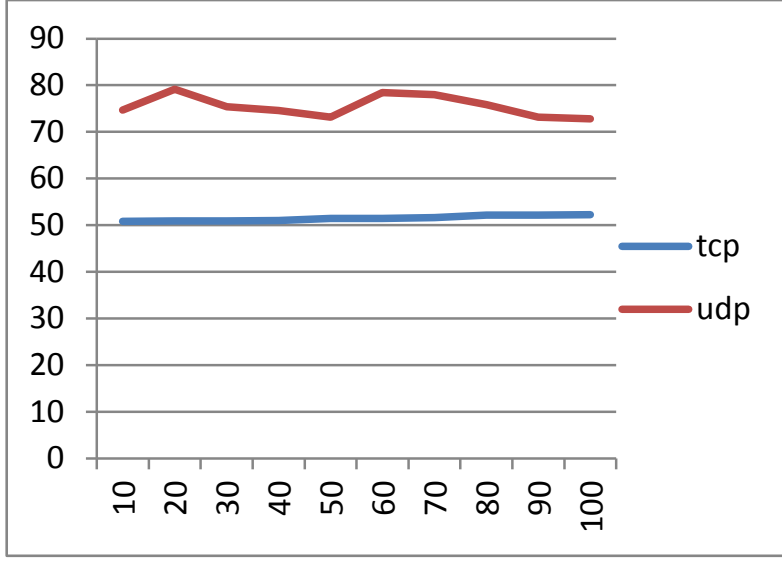

Figure 3: Packet delivery ratio versus pause time

This line graph illustrates the average packet delivery ratio over pause time by using TCP and UDP as the traffic modes.

\subsection{Total Packet Dropped}

The total number of packet dropped is calculated by subtracting the number of packet send to number of packet received.

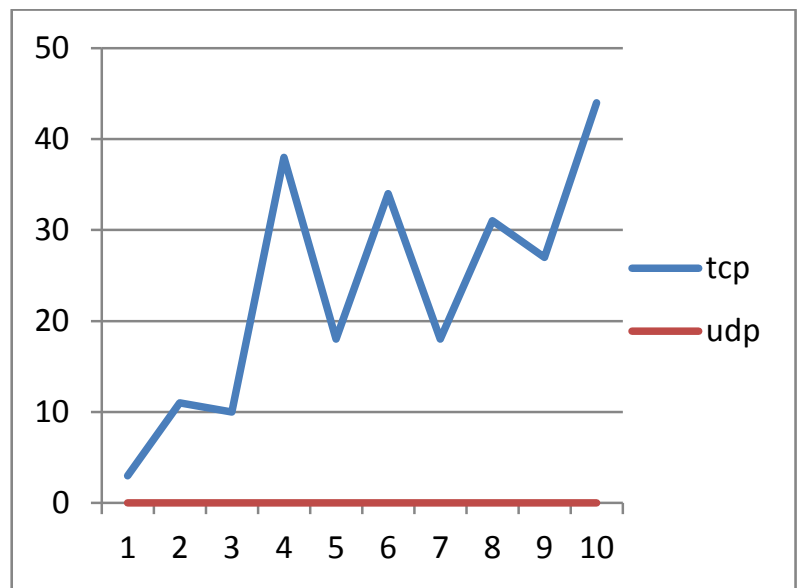

Figure 4: Total Packet Dropped versus pause time

This line graph illustrates the total packet dropped during simulation over pause time by using TCP and UDP as the traffic modes.

\subsection{Throughput}

Throughput is the average rate of successful packets delivered over a communication channel. This data may be delivered over a physical or logical link, or pass through a certain network node. 


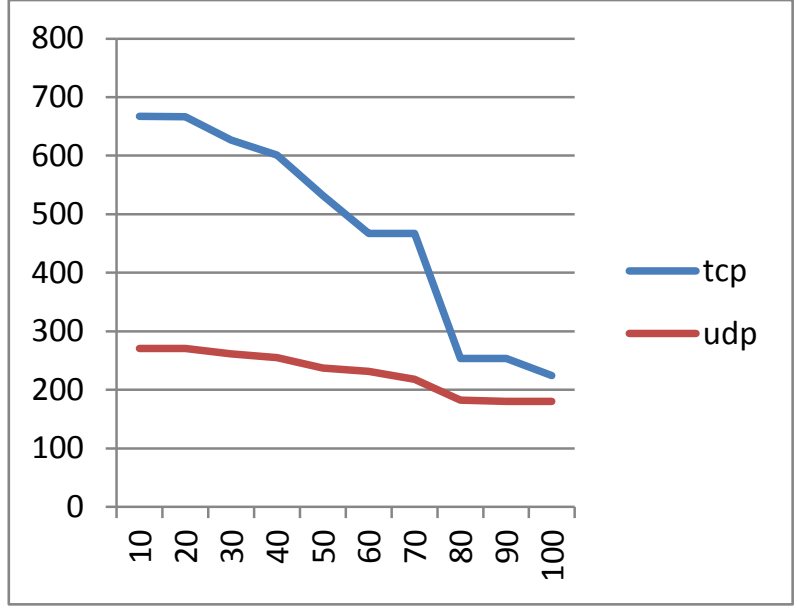

Figure 5: Throughput versus pause time

This line graph illustrates the average throughput over pause time by using TCP and UDP as the traffic modes.

\section{CONCLUSIONS AND FUTURE WORK}

\subsection{Conclusion}

It is analyzed TCP and UDP protocols in the hybrid network with Mobile IP and analyzed some performance metrics. It was found that in case of throughput in Hybrid network, TCP outperformed UDP and performed better in the hybrid network.

\subsection{Future Work}

There can be a lot of options to explore in a hybrid network. One way is to implement various MANET protocols in the adhoc network, I have used DSDV, other protocols can also be used and then the output can be compared. I have used Mobile IP as the gateway between wired and wireless network. Other gateways can also be used to compare the performance of different gateway protocols.

\section{ACKNOWLEDGMENT}

I would like to express my deep and sincere gratitude to Mr. Akhilesh Kosta, who guided me all the way in the research, and sparks this research with his constant help, tremendous efforts, and precious enlightenment.

Finally, special thanks must go to my husband and my family, who supported me with their love and encouragement, to do my best in life.

\section{REFERENCES}

[1] Perkins Charles E., May 1997, Mobile IP, IEEE Communications Magazine, Sun Microsystems

[2] Stevens W. R., TCP/IP Illustrated, Volume 1: The protocols. New York:Addison-Wesley, 1994..

[3] Schmid Andreas and Steigner, 2001: Avoiding Counting to Infinity in Distance vector Routing, Kluwer Academic Publishers, and Netherlands.

[4] Pentikousis H. Badr,2002, A survey of TCP in wiredcum-wireless environments, State University of New York at Stony Brook, IEEE COMMUNICATIONS, The Electronic Magazine of Original Peer-Reviewed Survey Articles

[5] Paul Rajashree, 2002,SELECTIVE-TCP FOR WIRED/WIRELESS NETWORKS, University of Kalyani.

[6] http://www.isi.edu/nsnam/ns/tutorial/nsscript6.html\#first

[7] Yu Wang, Weizhao Wang, Teresa A. Dahlberg, Truthful Routing for Wireless Hybrid Networks, Department of Computer Science

[8] W. R. Stevens, 1994, TCP/IP Illustrated, Volume 1: The Protocols Addison-Wesley.

[9] Mobile Communications Design Fundamentals John Wiley and Sons, W. C. Lee Y. 1993, 2nd edition.

[10] Tsaoussidis V., Badr H., Pentikousis K., Ge X., July 2000. "Energy/Throughput Tradeoffs of TCP Error Control Strategies", in Proceedings of IEEE Symposium on Computers. 